\title{
Measurement Uncertainty Evaluation using Monte Carlo Method based on LabVIEW
}

\author{
Wu Shilin ${ }^{a}$, Li Yuanqing ${ }^{b}$ and Fang Sui ${ }^{\mathrm{c}}$ \\ Department of Instrument Science and Technology, College of Mechatronics and Automation, \\ National University of Defense Technology, Changsha, Hunan, P. R. China, 410073 \\ a gfkdwsl@sina.com, ${ }^{b}$ lyqing.321@hotmail.com, ${ }^{c} 18684657642 @ 163 . c o m$
}

Keywords: measurement uncertainty, Monte Carlo method, LabVIEW, sample of input quantity Abstract. Monte Carlo method (MCM) for evaluation of measurement uncertainty is a beneficial supplement to the "Guide to the expression of uncertainty in measurement" (GUM). This paper introduces the method and the steps of measurement uncertainty evaluation by MCM based on LabVIEW, and an example is given out to validate the effect of measurement uncertainty evaluation by Monte Carlo method.

\section{Introduction}

In 2008, Joint Committee for Guides in Metrology (JCGM) issued a Supplement 1 to the "Guide to the expression of uncertainty in measurement" (GUM) - Propagation of distributions using a Monte Carlo method (MCM). The present Supplement 1 is intended to give added value to the GUM by providing guidance on aspects of uncertainty evaluation that are not explicitly treated in the GUM [1].

A mathematical tool or a programming software is required while using MCM to calculate the measurement uncertainty. Reference [2, 3, and 4] introduced the way to evaluate measurement uncertainty by MCM based on MATLAB. Reference [5,6] introduced the measurement uncertainty evaluation of monitor energy efficiency and total sulphur in coal by MCM based on Mathcad respectively. Reference [7] used Visual Basic to calculate measurement uncertainty by MCM. Reference [8] introduced the steps of measurement uncertainty evaluation by MCM based on Crystal Ball.

Those software introduced above have relatively higher requirements to measuring technical personnel, while LabVIEW has relatively lower requirements for its graphical programming. References $[9,10]$ have used LabVIEW to calculate the measurement uncertainty by GUM. This paper introduces the method and the steps of measurement uncertainty evaluation by MCM based on LabVIEW, which is significant to measuring technical personnel for calculating the measurement uncertainty by MCM.

\section{Monte Carlo method (MCM)}

MCM is a general numerical approach for carrying out the calculations required as part of an evaluation of measurement uncertainty. The main stages of MCM are as follows [1].

1) Determine the input quantities $X_{1}, \ldots, X_{N}$, the output quantity $Y$, and develop a model relating $Y$ and $X$, that is $Y=f\left(X_{1}, \ldots, X_{N}\right)$.

2) On the basis of available knowledge assign PDFs - Gaussian (normal), rectangular (uniform), etc. - to the $X_{i}$, and select the number $M$ of Monte Carlo trials.

3) Generate $M$ vectors, by sampling from the assigned PDFs, as realizations of the (set of $N$ ) input quantities $X_{i}$, for each such vector, form the corresponding model value of $Y$, yielding $M$ model values, that is

$$
y_{r}=f\left(x_{1 r}, x_{2 r}, \mathrm{~L}, x_{N r}\right), r=1, \ldots, M
$$


4) Sort these $M$ model values into strictly increasing order, using the sorted model values to provide a discrete representation $\mathrm{G}$ of the distribution function $G_{Y}(\eta)$ for the output quantity $Y$.

5) Use $\mathrm{G}$ to form an estimate $y$ of $Y$, the standard uncertainty $u(y)$ associated with $y$, and an appropriate coverage interval $\left[y_{\text {low }}, y_{\text {high }}\right]$ for $Y$, for a stipulated coverage probability $p$.

\section{Sampling from the input quantities}

Rectangular distributions $\mathbf{R}(\boldsymbol{a}, \boldsymbol{b})$. To sample from $\mathrm{R}(a, b)$, make a draw $r$ from the standard rectangular distribution $\mathrm{R}(0,1)$, and form

$$
\xi=a+(b-a) r
$$

Where, $a$ is the lower limit of the rectangular distribution, and $b$ is the upper limit of the rectangular distribution.

In LabVIEW, the block diagram of sampling from rectangular distributions shows as Fig. 1.

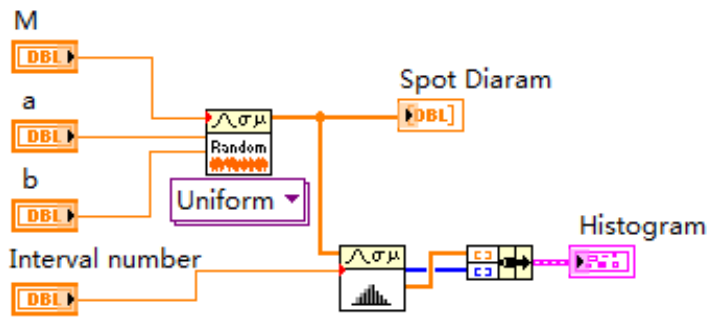

Figure 1. Sampling from rectangular distributions

Trapezoidal distributions Trap $(\boldsymbol{a}, \boldsymbol{b}, \boldsymbol{\beta})$. To sample from Trap $(a, b, \beta)$, make two draws $r_{1}$ and $r_{2}$ independently from the standard rectangular distribution $\mathrm{R}(0,1)$, and form

$$
\xi=a+\frac{(b-a)}{2}\left[(1+\beta) r_{1}+(1-\beta) r_{2}\right]
$$

Where, $a$ is the lower limit of the trapezoidal distribution, $b$ is the upper limit of the trapezoidal distribution, and $\beta$ equal to the ratio of the semi-width of the top of the trapezoid to that of the base.

In LabVIEW, the block diagram of sampling from triangular distributions shows as Fig. 2.

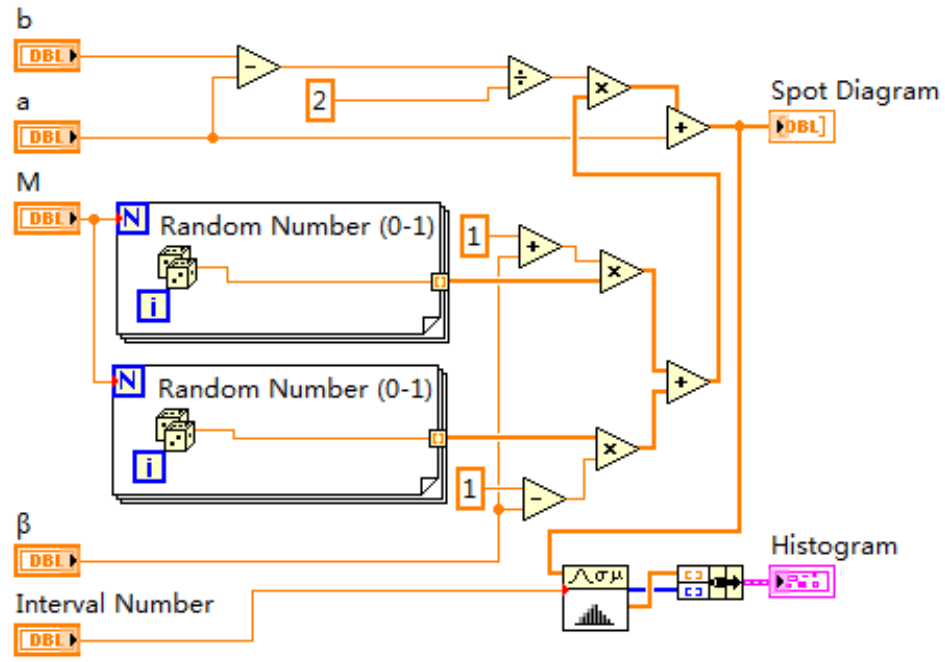

Figure 2. Sampling from trapezoidal distributions 
Triangular distributions $\mathbf{T}(\boldsymbol{a}, \boldsymbol{b})$. To sample from $\mathrm{T}(a, b)$, make two draws $r_{1}$ and $r_{2}$ independently from the standard rectangular distribution $\mathrm{R}(0,1)$, and form

$$
\xi=a+\frac{(b-a)}{2}\left(r_{1}+r_{2}\right)
$$

Where, $a$ is the lower limit of the triangular distribution, and $b$ is the upper limit of the triangular distribution.

In LabVIEW, the block diagram of sampling from triangular distributions shows as Fig. 3.

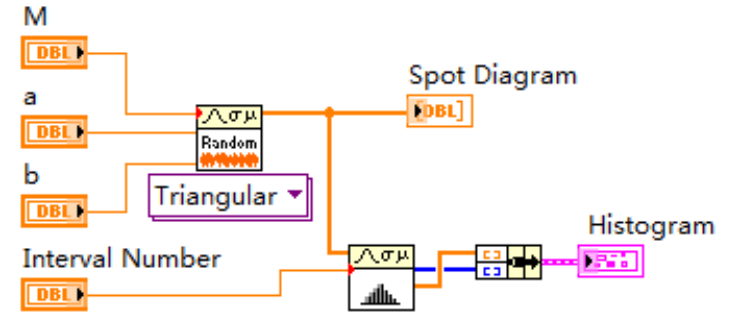

Figure 3. Sampling from triangular distributions

Arcsine distributions $\mathbf{U}(\boldsymbol{a}, \boldsymbol{b})$. To sample from $\mathrm{U}(a, b)$, make a draw $r$ from the standard rectangular distribution $\mathrm{R}(0,1)$, and form

$$
\xi=\frac{a+b}{2}+\frac{b-a}{2} \sin (2 \pi r)
$$

Where, $a$ is the lower limit of the Arcsine distribution, and $b$ is the upper limit of the Arcsine distribution.

In LabVIEW, the block diagram of sampling from Arcsine distributions shows as Fig. 4.

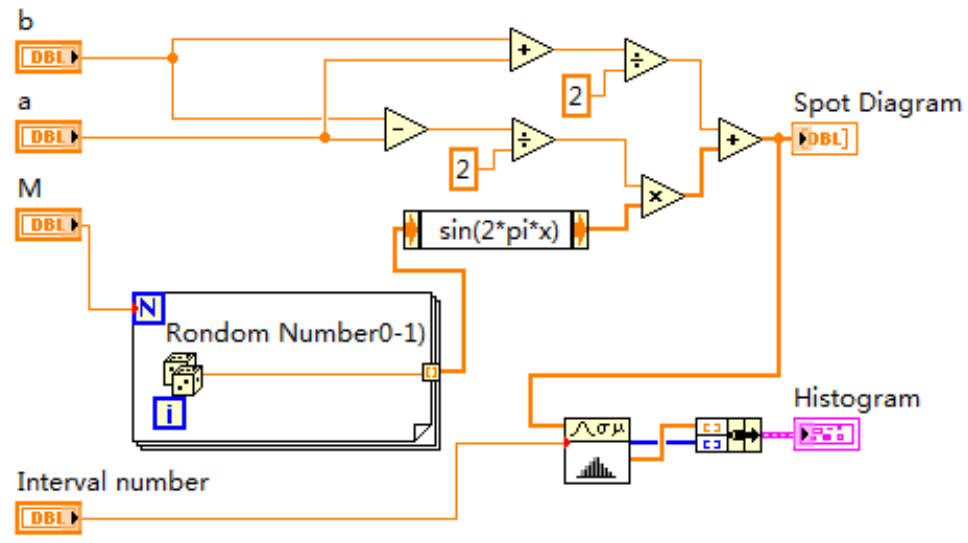

Figure 4. Sampling from Arcsine distributions

Gaussian distributions $\mathrm{N}\left(x, u^{2}(x)\right)$. To sample from $\mathrm{N}\left(x, u^{2}(x)\right)$, make a draw $r$ from the standard Gaussian distribution $\mathrm{N}(0,1)$, and form

$$
\xi=x+u(x) r
$$

Where, $x$ is the mean value of $X$, and $u(x)$ is the standard uncertainty of $X$.

In LabVIEW, the block diagram of sampling from Gaussian distributions shows as Fig. 5. 


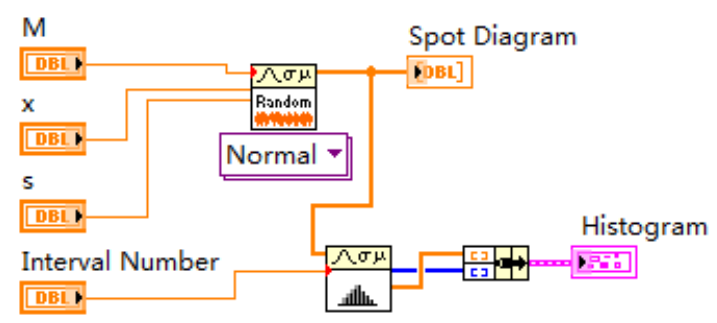

Figure 5. Sampling from Gaussian distributions

t-distributions $t_{v}\left(\bar{x}, u^{2}(x) / n\right)$. To sample from $t_{v}\left(\bar{x}, u^{2}(x) / n\right)$, make a draw $r$ from the central t-distribution $t_{v}$ with $v=n-1$ degrees of freedom, and form

$$
\xi=\bar{x}+\frac{u(x)}{\sqrt{n}} r
$$

Where, $\bar{x}$ is the mean value of $X$, and $u(x)$ is the standard uncertainty of $X$.

In LabVIEW, the block diagram of sampling from t-distributions shows as Fig. 6.

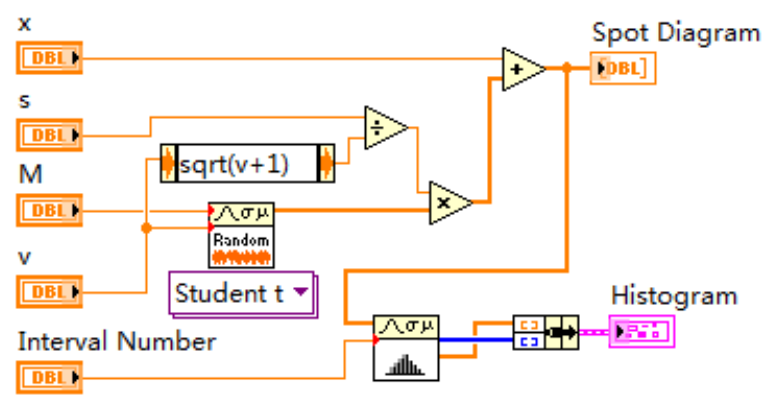

Figure 6. Sampling from t-distributions

Exponential distributions $E_{x}(1 / x)$. To sample from $E_{x}(1 / x)$, make a draw $r$ from the standard rectangular distribution $\mathrm{R}(0,1)$, and form

$$
\xi=-x \ln r
$$

Where, $x$ is the best estimate of $X$.

In LabVIEW, the block diagram of sampling from exponential distributions shows as Fig. 7.

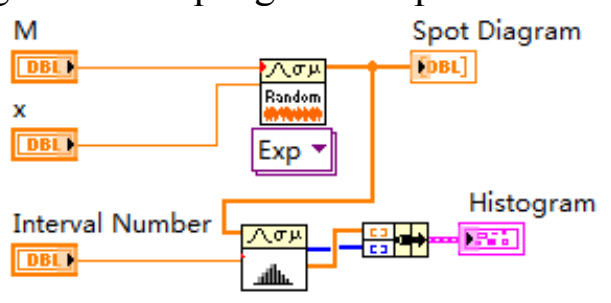

Figure 7. Sampling from exponential distributions

\section{Creating sub VIs for the sampling program}

After we have built some sampling programs, we can use them in another VI. A VI called from the block diagram of another VI is called a sub VI. A sub VI node corresponds to a subroutine call in text-based programming languages. To create a sub VI, we need to create an icon and build a connector pane. This paper create sub VIs from existing VIs (Fig. 1 to Fig. 7), and use Gaussian distribution (Fig. 5) as an example to introduce the creating of sub VIs. 
Creating an icon. Every VI displays an icon, shown as Fig. 8a, in the upper right corner of the front panal and block diagram windows. We can use the Icon Editor dialog box to edit the icon, double-click the icon in the upper right corner of the front panal or block diagram windows to display the Icon Editor dialog box, and modify the icon of the Gaussian distribution as Fig. 8b.

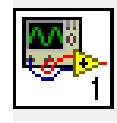

a) Default VI icon

$$
\text { Gau. }
$$

b) Modified VI icon

Figure 8. Icon of VI

Creating a connector pane. The connector pane is a set of terminals that corresponds to the controls and indicators of that VI, similar to the parameter list of a function call in text-based programming languages.

The connector pane of the Gaussian distribution VI (Fig. 5) shows as Fig. 9. Each rectangle on the connector pane represents a terminal. The left four terminals are inputs, they are the number $M$ of Monte Carlo trials, the best estimate value $x$, the standard uncertainty $u(x)$, and the interval number of histogram. The right two terminals are outputs, they are the sampled values and the histogram of the output.

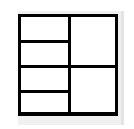

Figure 9. Connector pane of the Gaussian distribution VI

\section{Example of the application of MCM}

Using the calibration of digital multimeter (DMM) as an example, to calibrate its $100 \mathrm{~V}$ DC, GUM and MCM are used to evaluate the measurement uncertainty respectively.

The error of indication $E$ of the DMM to be calibrated is [11]:

$$
E=V_{i}-V_{s}+\Delta V_{i}-\Delta V_{s}
$$

Where, $V_{i}$ is the measurement result of the DMM, $V_{s}$ is the output of the muti-product calibrator, $\Delta V_{i}$ is the effect of the resolutio of the DMM, and $\Delta V_{s}$ is the effect of other factors to the muti-product calibrator, including instrument drift, instrument bias, nonlinear, gain variation, working temperature, supply voltage, and load effet caused by input impedance etc.

The uncertainty components are listed in Table 1.

Table 1. Uncertainty components

\begin{tabular}{c|c|c|c|c}
\hline $\begin{array}{c}\text { Influence } \\
\text { quantity }\end{array}$ & Estimate value [V] & $\begin{array}{c}\text { Standard } \\
\text { uncertainty [V] }\end{array}$ & PDF distributions & $\begin{array}{c}\text { Sensitivity } \\
\text { coefficient }\end{array}$ \\
\hline$V_{i}$ & 100.1 & 0 & - & 1 \\
\hline$V_{s}$ & 100.0 & 0.001 & Gaussian distribution & -1 \\
\hline$\Delta V_{i}$ & 0 & 0.029 & rectangular distribution & 1 \\
\hline$\Delta V_{s}$ & 0 & 0.0064 & rectangular distribution & -1 \\
\hline$E$ & 0.1 & - & - & - \\
\hline
\end{tabular}




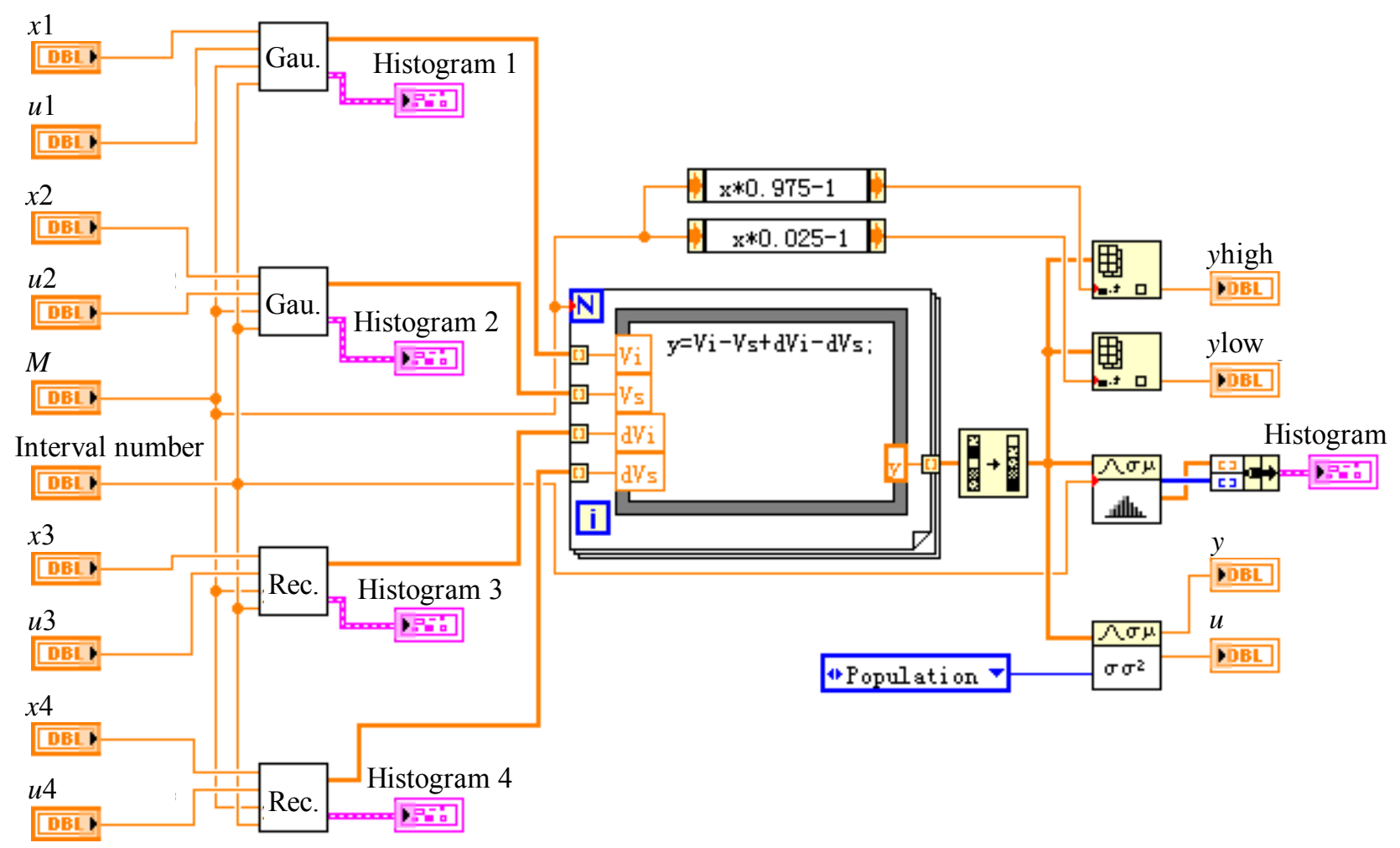

Figure 10. The block diagram of MCM

According to Eq. 9, the input quantities, the output quantity and the model relating $Y$ and $X$ are given out. According to Table 1, the distrubitions of input quantities are confirmed. Base on these known conditions, a block diagram is programmed (Fig. 10). The corresponding front panels of the output quantity show as Fig. 11 . The results are listed in Table 2, where the number $M$ of Monte Carlo trials is 1000000 . The results of GUM and that of reference [11] are also listed in Table 2. According to Fig. 11 and Table 2, it is obvious that the results of GUM don't confirm to the objective laws, and the results of MCM are more sentific.

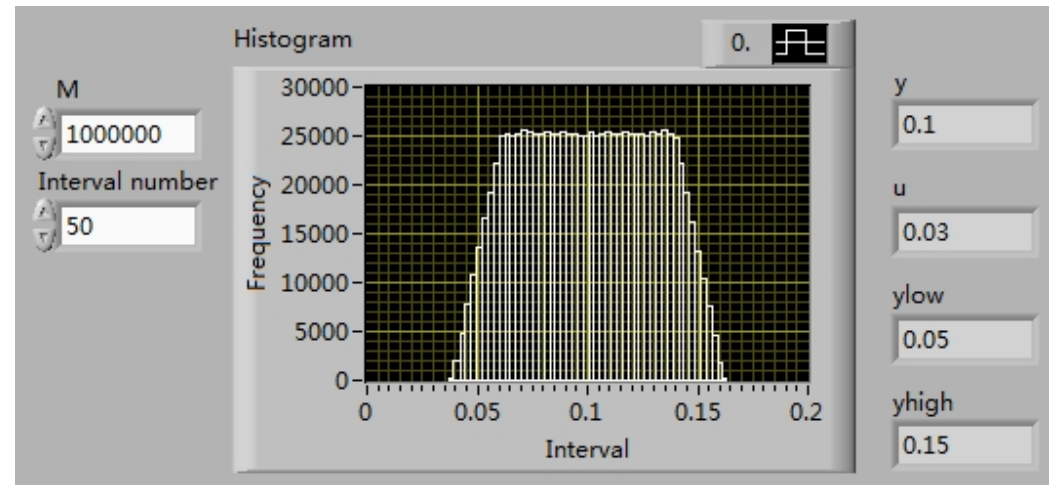

Figure 11. Front panel of the output quantity

Table 2. The results of different methods

\begin{tabular}{c|c|c|c|c|c}
\hline Method & Mean & Standard uncertainty & Distribution & $\begin{array}{c}\text { Coverage } \\
\text { factor }\end{array}$ & $\begin{array}{c}95 \% \text { coverage } \\
\text { interval }\end{array}$ \\
\hline GUM & 0.1 & 0.03 & Gaussian & 1.96 & {$[0.04,0.16]$} \\
\hline Reference [11] & 0.1 & 0.03 & rectangular & 1.65 & {$[0.05,0.15]$} \\
\hline MCM & 0.1 & 0.15 & trapezoidal & - & {$[0.05,0.15]$} \\
\hline
\end{tabular}




\section{Summary}

MCM can be applied include those in which the contributory uncertainties are not of approximately the same magnitude, or it is difficult or inconvenient to provide the partial derivatives of the model, as needed by the law of propagation of uncertainty, or the PDF for the output quantity is not a Gaussian distribution or a scaled and shifted t-distribution, or an estimate of the output quantity and the associated standard uncertainty are approximately of the same magnitude, or the models are arbitrarily complicated, and or the PDFs for the input quantities are asymmetric.

This paper introduces the method and the steps of measurement uncertainty evaluation by MCM based on LabVIEW. The operating process is simple, the interface is intuitive, and the uncertainty evaluation results are reliable.

\section{References}

[1] ISO/IEC. Evaluation of measurement data - Supplement 1 to the "Guide to the expression of uncertainty in measurement" - Propagation of distributions using a Monte Carlo method, JCGM 101: 2008.

[2] Zhou Taogeng. Monte Carlo method for evaluation of measurement uncertainty [M]. Peking: China ZHIJIAN publication house, 2013

[3] Liu Cuncheng, Hu Chang. Monte Carlo method for evaluation of measurement uncertainty in MATLAB [M]. Peking: China ZHIJIAN publication house, and Standard press of China, 2014

[4] Chen Huaiyan, Cao Yun, Han Jie. Evaluation of uncertainty in measurement based on a Monte Carlo method [J]. Journal of Electronic Measurement and Instrument, 2011, 25(4): pp. 301-308

[5] Hang Chenzhe, Xu Dinghua, and Cui Weiqun. Measurement uncertainty evaluation of monitor energy efficiency using Monte Carlo method [J]. China Metrology, 2012, (12): pp. 72-74.

[6] Zhang Jing, Zhao Jing. Evaluation of uncertainty measurement for determination of total sulphur in coal by Monte Carlo method [J]. Goal Quality Technology, 2014, (3): pp. 29-32

[7] Sheng Keping, He Baolin, and Yang Weihao. Calculating uncertainty with confidence level by Monte Carlo method [J]. Shanghai measurement and testing, 2005, (5): pp. 11-16

[8] Li Jun. Measurement uncertainty evaluation using Monte Carlo method based on Crystal Ball software [J]. Computer CD Software and Applications. 2012, (14): pp. 104-106

[9] Wang Haowei, Chen Zhenlin, Cheng Shaocheng. Uncertainty evaluation of digital multimeter measurement and implementation in LabVIEW [J]. Foreign electronic measurement technology, 2010, 29(7): pp. $75-78$

[10] Wei Xiaofei, Zou Jian, and Li Shiping. Developing of a Software for Calculating type A uncertainty based on LabVIEW [J]. Electrical measurement \& instrumentation, 2006, 43(12): pp. 59-61.

[11] Wu Shilin, Zhang Qi. Error analysis and data processing [M]. Peking: Tsinghua University press, 2010: pp. 190-193. 